SLAC-PUB-8435

hep-ph/0004143

April, 2000

\title{
Recalculation of Proton Compton Scattering in Perturbative QCD "̈
}

\author{
T. Brooksin and L. Dixon \\ Stanford Linear Accelerator Center \\ Stanford University \\ Stanford, CA 94309
}

\begin{abstract}
At very high energy and wide angles, Compton scattering on the proton $(\gamma p \rightarrow$ $\gamma p$ ) is described by perturbative QCD. The perturbative QCD calculation has been performed several times previously, at leading twist and at leading order in $\alpha_{s}$, with mutually inconsistent results, even when the same light-cone distribution amplitudes have been employed. We have recalculated the helicity amplitudes for this process, using contour deformations to evaluate the singular integrals over the light-cone momentum fractions. We do not obtain complete agreement with any previous result. Our results are closest to those of the most recent previous computation, differing significantly for just one of the three independent helicity amplitudes, and only for backward scattering angles. We present results for the unpolarized cross section, and for three different polarization asymmetries. We compare the perturbative QCD predictions for these observables with those of the handbag and diquark models. In order to reduce uncertainties associated with $\alpha_{s}$ and the three-quark wave function normalization, we have normalized the Compton cross section using the proton elastic form factor. The theoretical predictions for this ratio are about an order of magnitude below existing experimental data.
\end{abstract}

Submitted to Physical Review D

${ }^{*}$ Research supported by Department of Energy contract DE-AC03-76SF00515

$\dagger$ Supported in part by a National Science Foundation graduate fellowship 


\section{Introduction}

Exclusive real Compton scattering on the proton, $\gamma p \rightarrow \gamma p$, is a promising arena for studying the short-distance structure of the proton. In the limit of large energy $\sqrt{s}$ and fixed scattering angle $\theta$ in the center-of-mass frame, the real Compton amplitude should factorize as the convolution of a perturbative hard scattering matrix element with a nonperturbative light-cone distribution amplitude [1]. The distribution amplitude is for the three valence quarks in the proton; it describes how their longitudinal momentum is partitioned when their transverse separation is very small. Contributions of Fock space states with more partons in the proton's light-cone wave function should be suppressed by additional powers of $s$. However, the energy at which this asymptotic prediction of perturbative QCD (PQCD) becomes valid is not known a priori. Soft mechanisms such as the soft overlap (or handbag) model [2, 3, 4] and the diquark model [5, 6] could be comparable to, or even dominant over, the PQCD mechanism at the presently accessible center-of-mass energies of 2-4 GeV.

The PQCD prediction for $\gamma p \rightarrow \gamma p$ contains a number of uncertainties. First, only the Born level has been computed; next-to-leading-order corrections are likely to be large. Related to this, the Born level prediction is proportional to a high power of the running strong coupling constant, $\left[\alpha_{s}(\mu)\right]^{4}$, and its renormalization-scale $(\mu)$ dependence leads to a large normalization uncertainty on the cross section. Second, the form of the proton distribution amplitude is not well understood. Several groups have produced model distribution amplitudes based primarily on QCD sum rule analyses [7, 8, 9, 10, 11, 12, 13. These distribution amplitudes can lead to quite different predictions for the Compton helicity amplitudes. Most of the proposed distribution amplitudes tend to peak in a region where two of the three quarks carry relatively small fractions $x$ of the proton longitudinal momentum. This has led to skepticism about the applicability of PQCD at accessible energies [14, 15, 2, 3], because relatively soft sub-processes (relative to $\sqrt{s}$ ) can reorient quarks with small $x$ from the initial proton direction to the final proton direction.

Despite all these caveats, it is still useful to know the PQCD predictions for $\gamma p \rightarrow \gamma p$, if nothing else as an asymptotic limit. There have already been four separate calculations at Born level [16, 17, 18, 19]. However, no two results agree with each other, even when the same proton distribution amplitudes are assumed. Given this discrepancy in the literature, and the need for consistent predictions from the PQCD mechanism, we undertook an independent recalculation of this process. Our results in fact differ from all previous work, although we find reasonable agreement with ref. [18] for a subset of the helicity amplitudes, and excellent agreement with ref. 19] for forward scattering angles.

Our results are timely in view of the experimental situation. For over twenty years, the highest energy wide-angle Compton data available have been from an experiment at Cornell [20] which investigated the energy range $4.6 \mathrm{GeV}^{2}<s<12.1 \mathrm{GeV}^{2}$. These data

appear to obey an approximate $d \sigma / d t \propto s^{-6}$ scaling law, as predicted by PQCD, although more precise data would be useful to confirm or refute this behavior. An experiment now underway at Jefferson Lab [21] should soon improve the errors on the unpolarized cross section and its $\theta$ - and $s$-dependence, in the same kinematic range as the Cornell experiment. This experiment also plans to measure a polarization asymmetry, the transfer of longitudinal 
polarization from the incoming photon to the outgoing proton, for at least one angle. We shall discuss this asymmetry further in section 3. An upgrade of the Jefferson Lab electron beam to $12 \mathrm{GeV}$ [22] would allow for the very important extension of this experiment to higher energies. The proposed ELFE facility [23] with a $25 \mathrm{GeV}$ electron beam would also be a natural place to perform higher energy Compton measurements.

The remainder of this paper is organized as follows. In section 2 we outline the calculation. In section 3 we present results for the unpolarized cross section and for some different polarization asymmetries. Section 4 contains our conclusions.

\section{Calculation}

Since the general PQCD calculational framework for the Compton process has been described previously, e.g. in ref. [18], we will be brief here. The leading-twist PQCD factorization of the helicity amplitude $\mathcal{M}_{h h^{\prime}}^{\lambda \lambda^{\prime}}$ for incoming (outgoing) photon helicity $\lambda\left(\lambda^{\prime}\right)$ and proton helicity $h\left(h^{\prime}\right)$ is given by

$$
\begin{gathered}
\mathcal{M}_{h h^{\prime}}^{\lambda \lambda^{\prime}}=\sum_{d, i} \int_{0}^{1} d x_{1} d x_{2} d x_{3} d y_{1} d y_{2} d y_{3} \delta\left(1-\sum_{j=1}^{3} x_{j}\right) \delta\left(1-\sum_{k=1}^{3} y_{k}\right) \\
\times \phi_{i}(\vec{x}) T_{i}^{(d)}\left(\vec{x}, h, \lambda ; \vec{y}, h^{\prime}, \lambda^{\prime}\right) \phi_{i}^{*}(\vec{y})
\end{gathered}
$$

where the vectors $\vec{x} \equiv\left(x_{1}, x_{2}, x_{3}\right)$ and $\vec{y} \equiv\left(y_{1}, y_{2}, y_{3}\right)$ represent the quark longitudinal momentum fractions; $i$ labels the independent three-valence-quark Fock states of the proton, with distribution amplitudes $\phi_{i}(\vec{x})$; and $d$ represents the sum over the diagrams that contribute to the hard-scattering amplitude $T_{i}$.

The distribution amplitude represents the three-valence-quark component of the proton's light-cone wave function, after the latter is integrated over transverse momenta up to a factorization scale $\mu$. (Moments of the distribution amplitude can also be defined via the matrix elements of appropriate local three-quark operators.) The distribution amplitude evolves logarithmically with $\mu$, but (as was also done in refs. [16, 17, 18, 19]) we shall neglect this evolution here. The full distribution amplitude for a positive-helicity proton is, in the notation of ref. [18],

$$
\left|p_{\uparrow}\right\rangle=\frac{f_{N}}{8 \sqrt{6}} \int_{0}^{1} d x_{1} d x_{2} d x_{3} \delta\left(1-\sum_{j=1}^{3} x_{j}\right) \sum_{i=1}^{3} \phi_{i}(\vec{x})|i ; \vec{x}\rangle,
$$

where

$$
\begin{aligned}
|1 ; \vec{x}\rangle & =\left|u_{\uparrow}\left(x_{1}\right) u_{\downarrow}\left(x_{2}\right) d_{\uparrow}\left(x_{3}\right)\right\rangle, \\
|2 ; \vec{x}\rangle & =\left|u_{\uparrow}\left(x_{1}\right) d_{\downarrow}\left(x_{2}\right) u_{\uparrow}\left(x_{3}\right)\right\rangle, \\
|3 ; \vec{x}\rangle & =\left|d_{\uparrow}\left(x_{1}\right) u_{\downarrow}\left(x_{2}\right) u_{\uparrow}\left(x_{3}\right)\right\rangle .
\end{aligned}
$$

The normalization constant $f_{N}$ can be determined from QCD sum rules or lattice QCD. We choose $f_{N}=5.2 \times 10^{-3} \mathrm{GeV}^{2}$ (as in refs. [18, 19]). Fermi-Dirac statistics, isospin and spin 
symmetry result in only one independent distribution amplitude, $\phi_{1}$; the other two are given by

$$
\begin{aligned}
& \phi_{2}\left(x_{1}, x_{2}, x_{3}\right)=-\phi_{1}\left(x_{1}, x_{2}, x_{3}\right)-\phi_{1}\left(x_{3}, x_{2}, x_{1}\right), \\
& \phi_{3}\left(x_{1}, x_{2}, x_{3}\right)=\phi_{1}\left(x_{3}, x_{2}, x_{1}\right) .
\end{aligned}
$$

In addition to neglecting evolution of the distribution amplitude, we shall also take $\alpha_{s}$ to be fixed. The Born-level cross section then scales as $\alpha_{s}^{4} \times f_{N}^{4}$.

The hard scattering amplitude is computed for three collinear incoming and outgoing quarks. The color and electric charge dependence can be factored off of each diagram as

$$
T_{i}^{(d)}\left(\vec{x}, h, \lambda ; \vec{y}, h^{\prime}, \lambda^{\prime}\right)=C^{(d)} g^{4} Z_{i}^{(d)} \tilde{T}^{(d)}\left(\vec{x}, \vec{y} ; h, \lambda, h^{\prime}, \lambda^{\prime}\right),
$$

where $C^{(d)}$ is the color factor, $g$ is the strong coupling constant, and $Z_{i}^{(d)}$ is the appropriate product of quark electric charges, while $\tilde{T}^{(d)}$ is color and flavor independent.

The helicities of the quarks in the hard scattering amplitude are conserved by the gauge interactions; therefore the proton helicity is conserved, and $\mathcal{M}_{h h^{\prime}}^{\lambda \lambda^{\prime}}=0$ for $h \neq h^{\prime}$. Parity and time-reversal invariance further reduce the number of independent helicity amplitudes to three, which we take to be

$$
\mathcal{M}_{\uparrow \uparrow}^{\uparrow \uparrow}, \quad \mathcal{M}_{\uparrow \uparrow}^{\uparrow \downarrow}, \quad \text { and } \mathcal{M}_{\uparrow \uparrow}^{\downarrow \downarrow}
$$

In principle, 378 diagrams contribute to the hard scattering amplitude. However, 42 of them contain three-gluon vertices and have a vanishing color factor. Many others vanish for individual helicity configurations.

We adopted the technique in ref. [18] of using the parity symmetry (denoted $\mathcal{E}$ therein) between certain classes of diagrams to reduce the number that had to be computed, while reserving the time-reversal symmetry as a check. All diagrams were computed by two independent computer programs, both based on the formalism outlined in ref. [24]. These expressions were found to be identical to those used in the two most recent computations [18, 19]. PThus we agree completely with refs. [18, 19] on the hard scattering amplitude $T_{i}$.

The next step is to perform the four-dimensional integration in eq. (1) over the independent quark momentum fractions. For the various model distribution amplitudes [7, 8, 9, 10] we used the coefficients of $\phi_{1}$ listed in Table I of ref. [18 (and eq. (6) of ref. [11]). Many diagrams include denominators that vanish inside the $(\vec{x}, \vec{y})$ integration region, due to the presence of an internal quark and/or gluon that can go on shell. This is not a true longdistance singularity, and all the integrals are finite, diagram by diagram, but it is a technical obstacle to obtaining a reliable value for the integral. In the notation of ref. [18], the Feynman $i \varepsilon$ prescription leads to singular denominators of the form

$$
\frac{1}{(x, y)+i \varepsilon}=\mathrm{P} \frac{1}{(x, y)}-i \pi \delta((x, y)),
$$

\footnotetext{
${ }^{\ddagger}$ We compared our results for each diagram to the formulae given in Tables III and IV of ref. [18]. These tables contain three errors (found by M. Vanderhaeghen [25] as well as us) in addition to one in diagram $A 71$ that was published in an erratum. However, all these errors are typographical and do not affect the numerical results in that paper [26]. The errors are: In the denominator of $\tilde{T}^{(A 44)}(x, \uparrow, \downarrow ; y, \uparrow, \downarrow),\left(\bar{x}_{3}, x_{1}\right)$ should be $\left(\bar{x}_{3}, y_{1}\right) ; \tilde{T}^{(C 75)}(x, \uparrow, \downarrow ; y, \uparrow, \downarrow)$ should be multiplied by $1 / c$; and the diagram related to $\overline{C 77}$ by $\mathcal{T} \circ \mathcal{E}$ should be $\overline{F 11}$, not $\overline{F 33}$.
} 
where $\mathrm{P}$ stands for principal part and $(x, y) \equiv x\left(1-y s^{2}\right)-y c^{2}$, with $s=\sin (\theta / 2)$ and $c=\cos (\theta / 2)$. Diagrams can be classified by the number of singular factors found in the denominator; for the Compton process this number can be $0,1,2$ or 3 . The presence of on-shell partons in the Born-level hard scattering amplitude (for particular values of $(\vec{x}, \vec{y})$ ) leads to large phases in the PQCD amplitude [17, 16, 18]. This is in contrast to the handbag model, which predicts an imaginary part that is small and beyond the accuracy of the model.

At least four different numerical methods have previously been applied to handle the singular integrations. Ref. [16] performed a Taylor expansion of the numerators of the integrand symmetrically about each singularity. Ref. [17] kept the $\varepsilon$ in eq. (7) explicit, and evaluated the integrals for a sequence of $\varepsilon$ values tending to zero, looking for stable results. Ref. 18 handled the imaginary parts of the singular integrals by solving the $\delta$ function constraint explicitly, and carried out the real, principal-part integrals by folding the region of integration over at the singularity, so that the integrand is manifestly finite. Finally, ref. [19] deformed the $(\vec{x}, \vec{y})$ integration contour into the complex plane, an elegant technique that requires relatively little bookkeeping for its implementation.

We adopted a variation of the contour deformation technique [19]. We first let

$$
\begin{array}{lll}
x_{1}=\xi_{1}, & x_{2}=\left(1-\xi_{1}\right)\left(1-\xi_{2}\right), & x_{3}=\left(1-\xi_{1}\right) \xi_{2}, \\
y_{1}=\eta_{1}, & y_{2}=\left(1-\eta_{1}\right)\left(1-\eta_{2}\right), & y_{3}=\left(1-\eta_{1}\right) \eta_{2},
\end{array}
$$

so that the four independent variables $\left(\xi_{1}, \xi_{2}, \eta_{1}, \eta_{2}\right)$ were integrated on the interval $[0,1]$. We then deformed the single variable $\eta_{1}$ into the complex plane, so that it ran either over the piecewise linear contour $0 \rightarrow i \epsilon \rightarrow 1+i \epsilon \rightarrow 1$, or over a semi-circular contour extending from 0 to 1 . Note that this simultaneously deforms both $y_{1}$ and $y_{3}$, towards opposite sides of the real axis, while $x_{1}$ and $x_{3}$ remain real. Inspection of the denominator factors in Tables III and IV of ref. [18 shows that this deformation is sufficient to correctly bypass the singularities in every Compton diagram. For example, the denominator of diagram A16 includes the factors $\left[\left(x_{1}, y_{1}\right)+i \varepsilon\right]\left[\left(\bar{x}_{3}, y_{1}\right)+i \varepsilon\right]\left[\left(y_{3}, x_{3}\right)+i \varepsilon\right]$, where $\bar{x}_{i} \equiv 1-x_{i}, \bar{y}_{i} \equiv 1-y_{i}$. Using the identity $(x, y)=(\bar{y}, \bar{x})$, the singular factors can be rewritten as $\left[\left(1-y_{1}, \bar{x}_{1}\right)+\right.$ $i \varepsilon]\left[\left(1-y_{1}, x_{3}\right)+i \varepsilon\right]\left[\left(y_{3}, x_{3}\right)+i \varepsilon\right]$, which shows that $y_{1}$ and $y_{3}$ should indeed be deformed in opposite directions. If the diagram happens to contain denominators of the form $\left(x_{i}, y_{1}\right)$ or $\left(y_{3}, x_{i}\right)$, instead of $\left(y_{1}, x_{i}\right)$ or $\left(x_{i}, y_{3}\right)$, as does diagram A16, then the imaginary part should be multiplied by an overall minus sign (or equivalently, the contour should be deformed in the opposite direction with respect to the real axis).

After making these contour deformations, the real and imaginary parts of the complex integrals were performed separately using the Monte Carlo integration routine VEGAS [27]. Two independent versions of the contour integration were implemented numerically, with two different choices of contour (piecewise linear vs. semi-circular), and we also varied the deformation parameter $\epsilon$, obtaining stable results.

A third version of the integration program was constructed, which employed the GaussLegendre formalism [28] with ten points per integration variable, instead of VEGAS. Although the Gauss-Legendre errors were larger than the VEGAS errors, the two sets of results were completely consistent with each other (and were both inconsistent with results from previous work; see section 3.1). 
We carried out further checks on our integration routines. For diagrams with only one singular factor in the denominator, one can integrate the imaginary part analytically. Using this procedure we checked the imaginary part of all diagrams with one singularity. One can also check the diagrams with no singularities in the same manner. A second check employed the identity

$$
\frac{1}{(x, y)(x, z)}=\frac{1-y s^{2}}{c^{2}(y-z)(x, y)}-\frac{1-z s^{2}}{c^{2}(y-z)(x, z)} .
$$

Using eq. (9) one can reduce all three-singularity diagrams to two singularities. (These expressions can be reduced no further, though, because the four remaining singular variables are all different.) One can also reduce all diagrams that initially had two singularities to one-singularity diagrams, allowing their imaginary parts to be computed analytically. Our integration techniques were robust against all of these tests.

Finally, Table V of ref. [18] gives detailed results for diagram A51, which has two denominator singularities. We agree completely with these results, for both the real and imaginary parts. We note that ref. [18 also attempted to evaluate this diagram by implementing the explicit $\varepsilon \rightarrow 0$ method of ref. [17], but they obtained very different results for the imaginary part, compared with the results of their folding method. Ref. [18] claims that the explicit $\varepsilon \rightarrow 0$ method is not numerically stable. Since we agree with their results for diagram A51, we do not have cause to disagree with this claim.

\section{Results}

\subsection{Comparison with previous work}

We computed the Compton helicity amplitudes for a variety of distribution amplitudes, which we refer to as CZ [7], GS [8], KS [9], COZ [10], HET [11], and ASY (the distribution amplitude for asymptotically large energy scales, $\left.\phi_{1}\left(x_{1}, x_{2}, x_{3}\right)=120 x_{1} x_{2} x_{3}\right)$. The CZ, $\mathrm{KS}$ and COZ distribution amplitudes, which satisfy the constraints imposed by QCD sum rules [7, 9], are qualitatively similar. They feature a peak in $\phi_{1}$ for $x_{1} \approx 1, x_{2,3} \approx 0$; that is, the $u$ quark with the same helicity as the proton carries most of the momentum. The GS distribution amplitude has a peak in $\phi_{1}$ for $x_{1,3} \approx 1 / 2, x_{2} \approx 0$; thus it splits the momentum more equitably between the two quarks carrying the proton's helicity. The HET distribution amplitude is intermediate in shape between GS and the $\{\mathrm{CZ}, \mathrm{KS}, \mathrm{COZ}\}$ class.

Before discussing our full results, we present a comparison of results in the literature. Here we choose the COZ distribution amplitude, since it was employed in four of the five existing calculations. (Only the earliest calculation [16], which was later superseded [17], did not use the COZ distribution amplitude.) The overall cross-section normalizations in the literature are sometimes difficult to determine, due for example to unspecified choices for $\alpha_{s}$. Therefore we choose to compare results for the following (normalization-independent) 


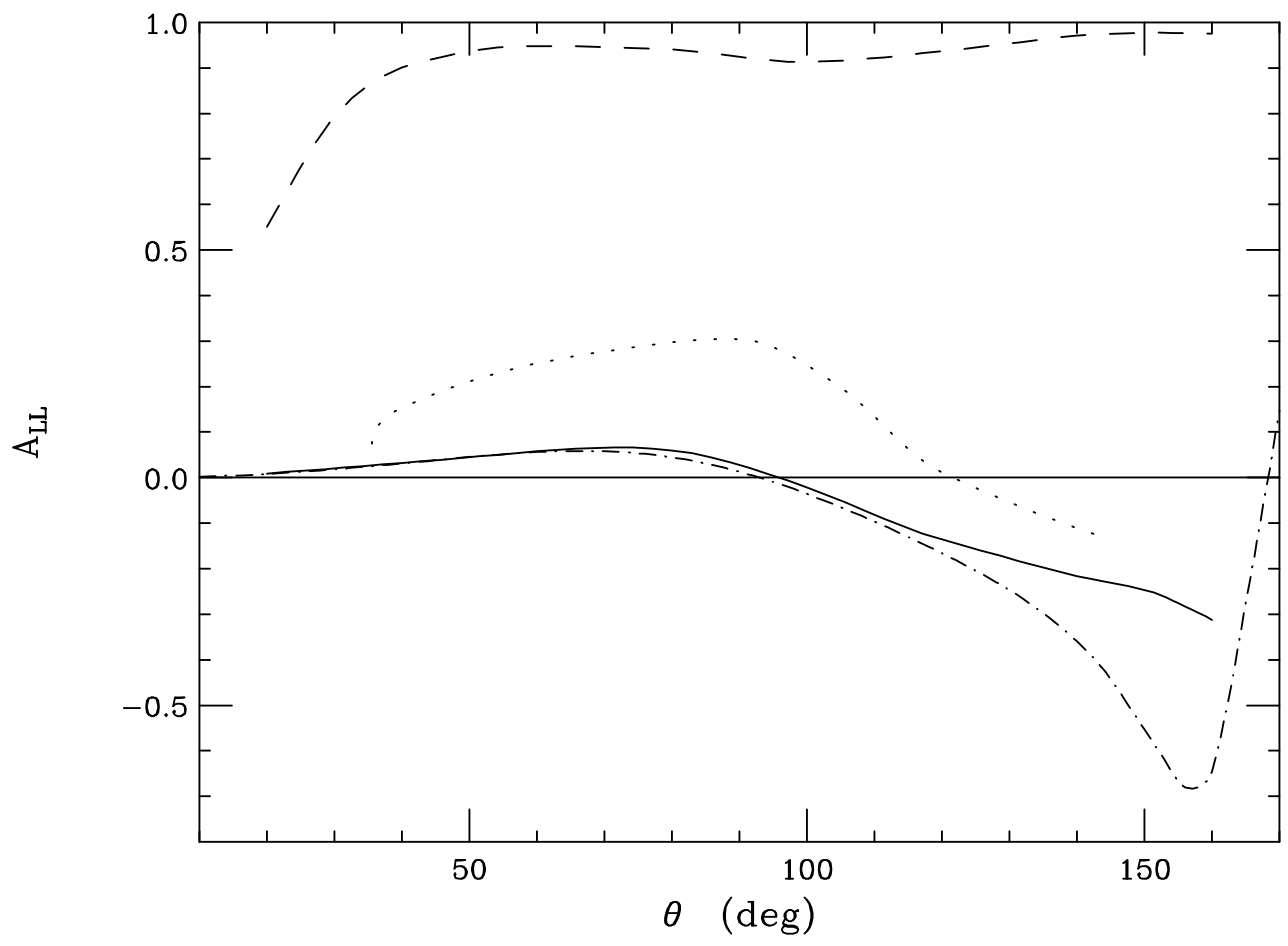

Figure 1: Four different calculations of the polarization asymmetry $A_{L L}$ defined in eq. (10), for the COZ distribution amplitude. The dotted line is from ref. [17], the dashed line from ref. [18], the dot-dash line from ref. [19], and the solid line from this work.

initial-state helicity correlation [19],

$$
A_{L L} \equiv \frac{\frac{d \sigma_{+}^{+}}{d t}-\frac{d \sigma_{+}^{-}}{d t}}{\frac{d \sigma_{+}^{+}}{d t}+\frac{d \sigma_{+}^{-}}{d t}}
$$

where $d \sigma_{h}^{\lambda} / d t$ is the differential cross section for a helicity $h$ proton scattering off a helicity $\lambda$ photon.

Figure 1 shows that none of the four calculations of $A_{L L}$ agrees completely with any other. The only two results that are very close are ours and that of ref. [19]. These two curves are in excellent agreement for $\theta<110^{\circ}$; however, we do not reproduce the prominent dip of ref. [19] in the backward region. This statement is true for the four distribution amplitudes we have compared: KS, COZ, CZ and ASY [19, 25]. Figure 14 of the second reference in [19] shows that the dip in $A_{L L}$ derives from $\frac{d \sigma}{d t}\left(\gamma_{\uparrow} p_{\uparrow} \rightarrow \gamma p\right) \propto\left|\mathcal{M}_{\uparrow \uparrow}^{\uparrow \uparrow}\right|^{2}+\left|\mathcal{M}_{\uparrow \uparrow}^{\uparrow \downarrow}\right|^{2}$, and not from $\frac{d \sigma}{d t}\left(\gamma_{\downarrow} p_{\uparrow} \rightarrow \gamma p\right) \propto\left|\mathcal{M}_{\uparrow \uparrow}^{\uparrow \downarrow}\right|^{2}+\left|\mathcal{M}_{\uparrow \uparrow}^{\downarrow \downarrow}\right|^{2}$. Indeed, we agree with their $\gamma_{\downarrow} p_{\uparrow} \rightarrow \gamma p$ cross section for all angles to better than 10\%, up to an overall normalization factor which can be accounted for by different choices for $\alpha_{s}$. We agree with the $\gamma_{\uparrow} p_{\uparrow} \rightarrow \gamma p$ cross section only for $\theta<110^{\circ}$, however. This suggests that the discrepancy with ref. [19 is predominantly from the single helicity amplitude $\mathcal{M}_{\uparrow \uparrow}^{\uparrow \uparrow}$. 
The curve from ref. [17] has the same general shape as ours, but is offset from it. The phases of the dominant helicity amplitudes given in ref. [17] actually agree quite well with our results in figs. 50 below; the magnitudes are offset by relatively angle-independent factors.

Ref. [18] finds a very large asymmetry. We have made a detailed comparison of our COZ results with those of ref. [18], for the real and imaginary parts of the three independent helicity amplitudes. Each amplitude has been further split into four pieces [26], according to the number of singular propagators in the diagram (as determined from Tables III and IV of ref. [18]). The zero propagator terms (which were integrated analytically by both groups) agree to high precision (6 digits). The one propagator terms agree to within VEGAS errors, except for the imaginary part of one helicity amplitude $\left(\mathcal{M}_{\uparrow \uparrow}^{\uparrow \downarrow}\right)$ which is within $10 \%$. For the two propagator terms, we are in agreement on the real part of $\mathcal{M}_{\uparrow \uparrow}^{\uparrow \uparrow}$ and $\mathcal{M}_{\uparrow \uparrow}^{\uparrow \downarrow}$, but have a large discrepancy in the imaginary part. Strangely enough, for $\mathcal{M}_{\uparrow \uparrow}^{\downarrow \downarrow}$ we agree on the imaginary part but disagree on the real part! For the three propagator terms, both the real and imaginary parts disagree for all three helicity amplitudes. The bulk of our overall numerical disagreement comes from the two propagator terms contributing to the imaginary part of $\mathcal{M}_{\uparrow \uparrow}^{\uparrow \uparrow}$. The two propagator terms are often 100 times larger than ours, and they drive ref. [18]'s values for $\operatorname{Im} \mathcal{M}_{\uparrow \uparrow}^{\uparrow \uparrow}$ to be roughly a factor of 10 larger than ours.

We also calculated $A_{L L}$ for the COZ distribution amplitude using Gauss-Legendre integration instead of VEGAS. The result agrees with our VEGAS result shown in fig. 1 (albeit with larger errors), and it disagrees with the other results, in particular that of ref. [19] for $\theta>110^{\circ}$.

\subsection{Helicity amplitudes and unpolarized cross section}

In figs. 24 we display our results for the polarized differential cross sections,

$$
s^{6} \frac{d \sigma_{h h^{\prime}}^{\lambda \lambda^{\prime}}}{d t}=\frac{s^{4}}{16 \pi}\left|\mathcal{M}_{h h^{\prime}}^{\lambda \lambda^{\prime}}\right|^{2}
$$

for the three independent helicity configurations. Each figure plots the results for five different distribution amplitudes. (For HET we shall only plot the unpolarized cross section.) These plots were made for $\alpha_{\mathrm{em}}^{-1}=137.036, \alpha_{s}=0.3$ and $f_{N}=5.2 \times 10^{-3} \mathrm{GeV}^{2}$, so they can be compared directly with ref. 18]. The phases of the helicity amplitudes are plotted in figs. 57; the GS distribution amplitude has a much different behavior and is therefore plotted separately, in fig. 8. The phases are generally large; indeed $\mathcal{M}_{\uparrow \uparrow}^{\uparrow \downarrow}$ is almost pure imaginary (except for the GS distribution amplitude). For reference, we also provide in Table 1 our numerical results for the real and imaginary part of $\mathcal{M}_{\uparrow \uparrow}^{\uparrow \uparrow}$, for the COZ distribution amplitude, including errors from the VEGAS integration.

Figure 9 shows our predictions for the unpolarized differential Compton cross section, given by

$$
s^{6} \frac{d \sigma}{d t}=\frac{1}{4} \sum_{\lambda, \lambda^{\prime}, h, h^{\prime}} s^{6} \frac{d \sigma_{h h^{\prime}}^{\lambda \lambda^{\prime}}}{d t},
$$

along with the experimental data from ref. [20]. For the values used $\alpha_{s}=0.3, f_{N}=$ $5.2 \times 10^{-3} \mathrm{GeV}^{2}$, the predictions lie at least an order of magnitude below the data. Since 


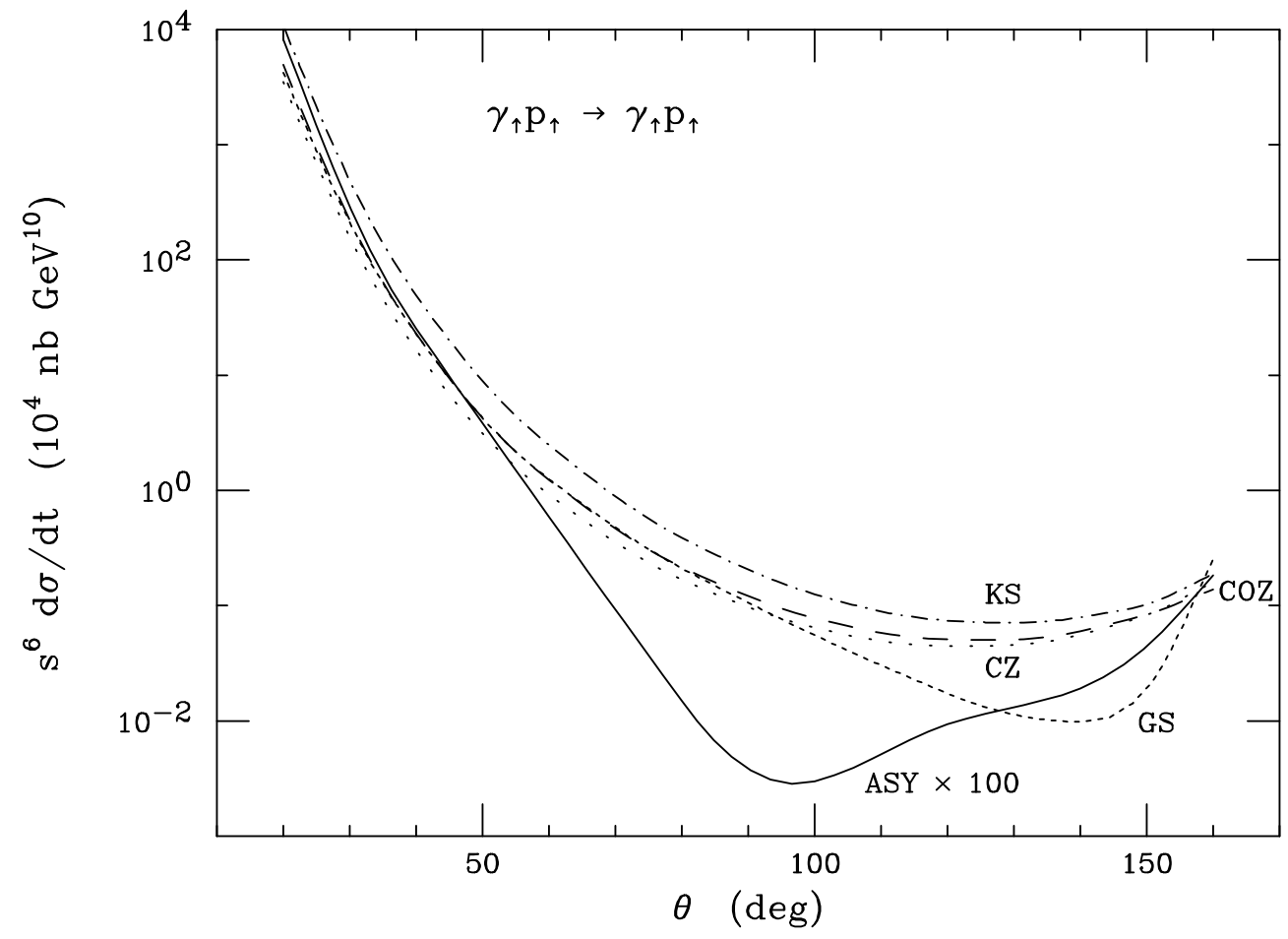

Figure 2: The cross section for $\gamma_{\uparrow} p_{\uparrow} \rightarrow \gamma_{\uparrow} p_{\uparrow}$ for five different distribution amplitudes, CZ, COZ, KS, GS and ASY. The results for the asymptotic distribution amplitude (ASY) have been multiplied by 100 . 


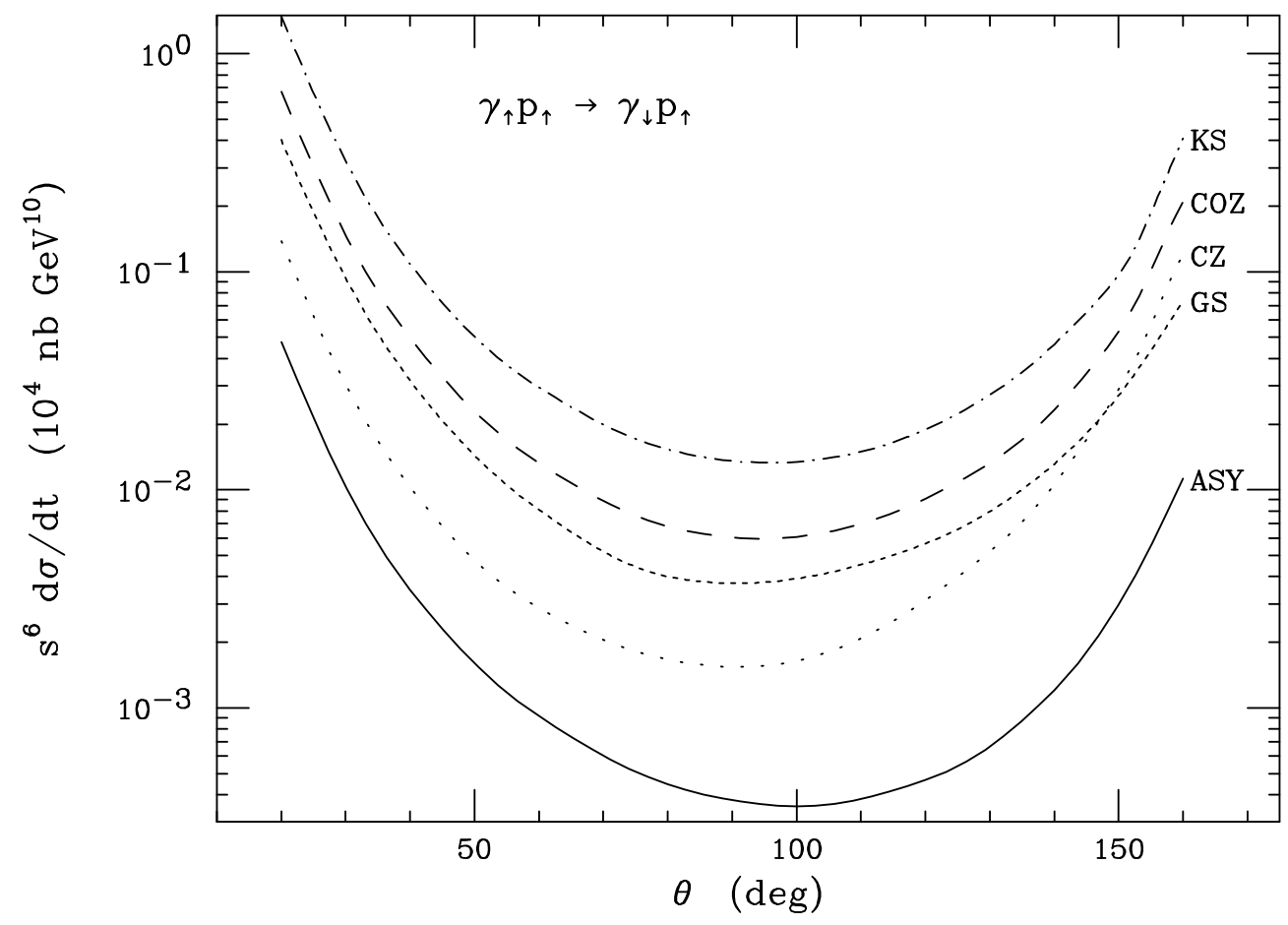

Figure 3: The cross section for $\gamma_{\uparrow} p_{\uparrow} \rightarrow \gamma_{\downarrow} p_{\uparrow}$.

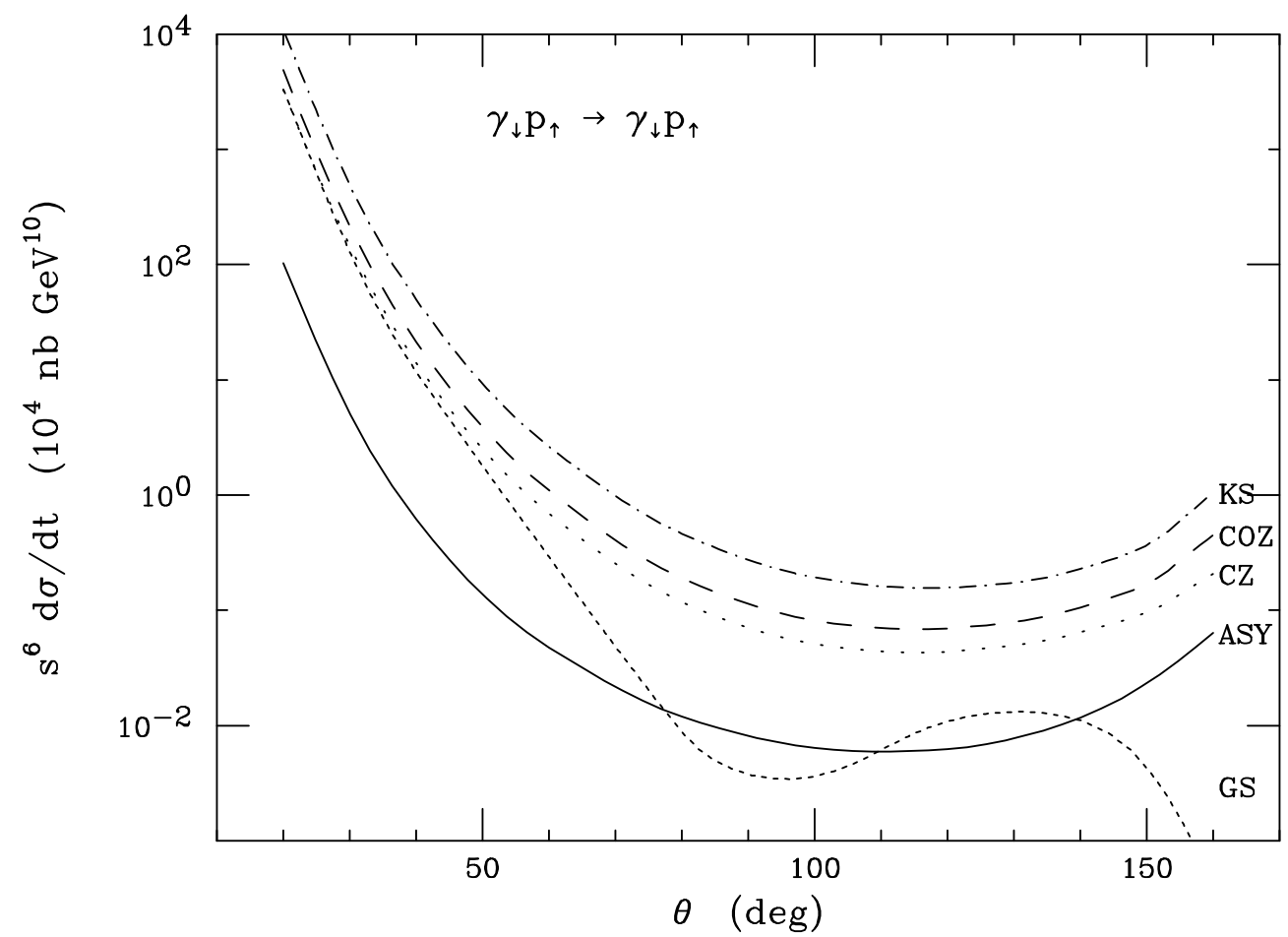

Figure 4: The cross section for $\gamma_{\downarrow} p_{\uparrow} \rightarrow \gamma_{\downarrow} p_{\uparrow}$. 


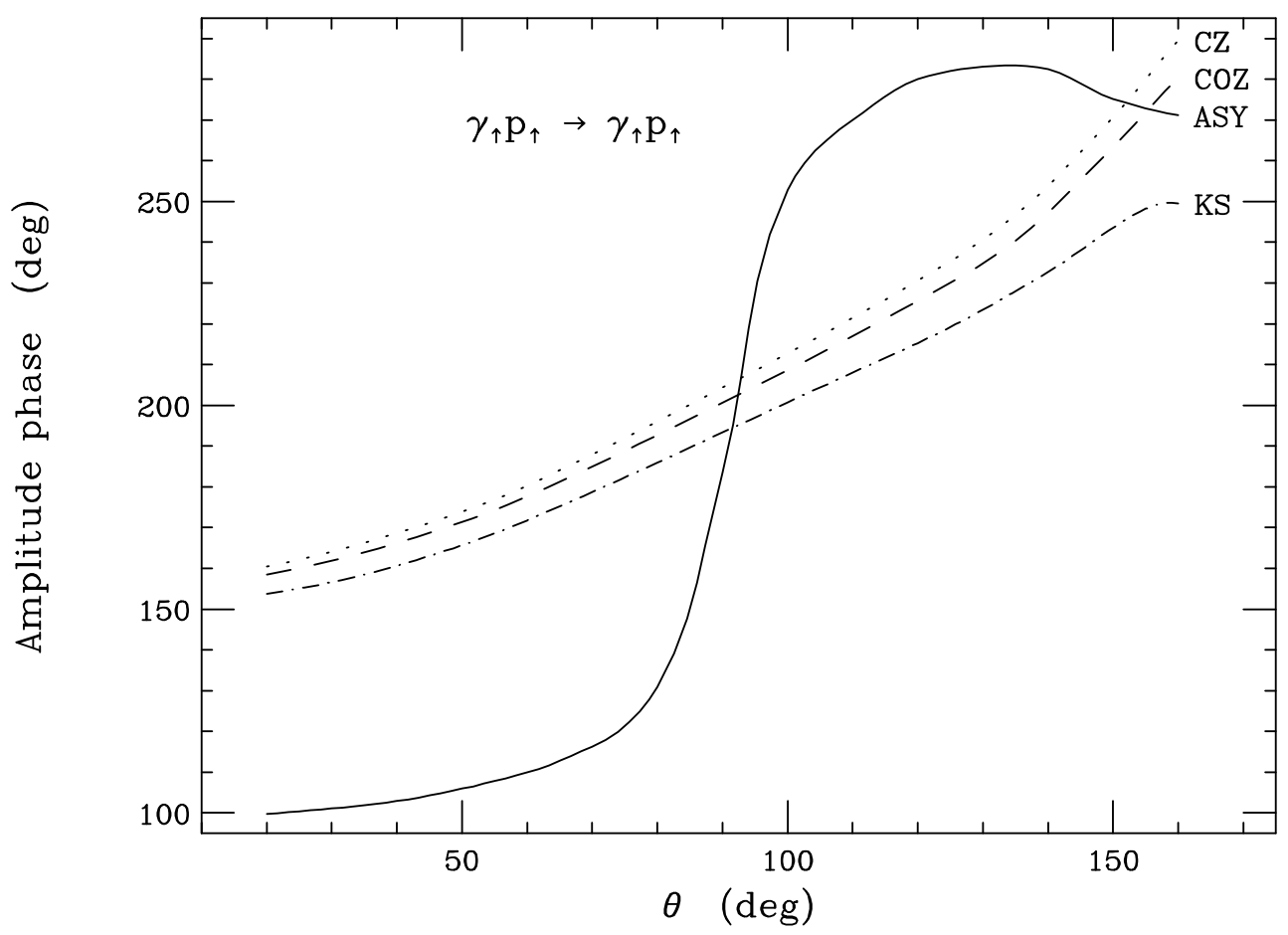

Figure 5: Phase of the helicity amplitude for $\gamma_{\uparrow} p_{\uparrow} \rightarrow \gamma_{\uparrow} p_{\uparrow}$ for the distribution amplitudes CZ, COZ, KS and ASY. 


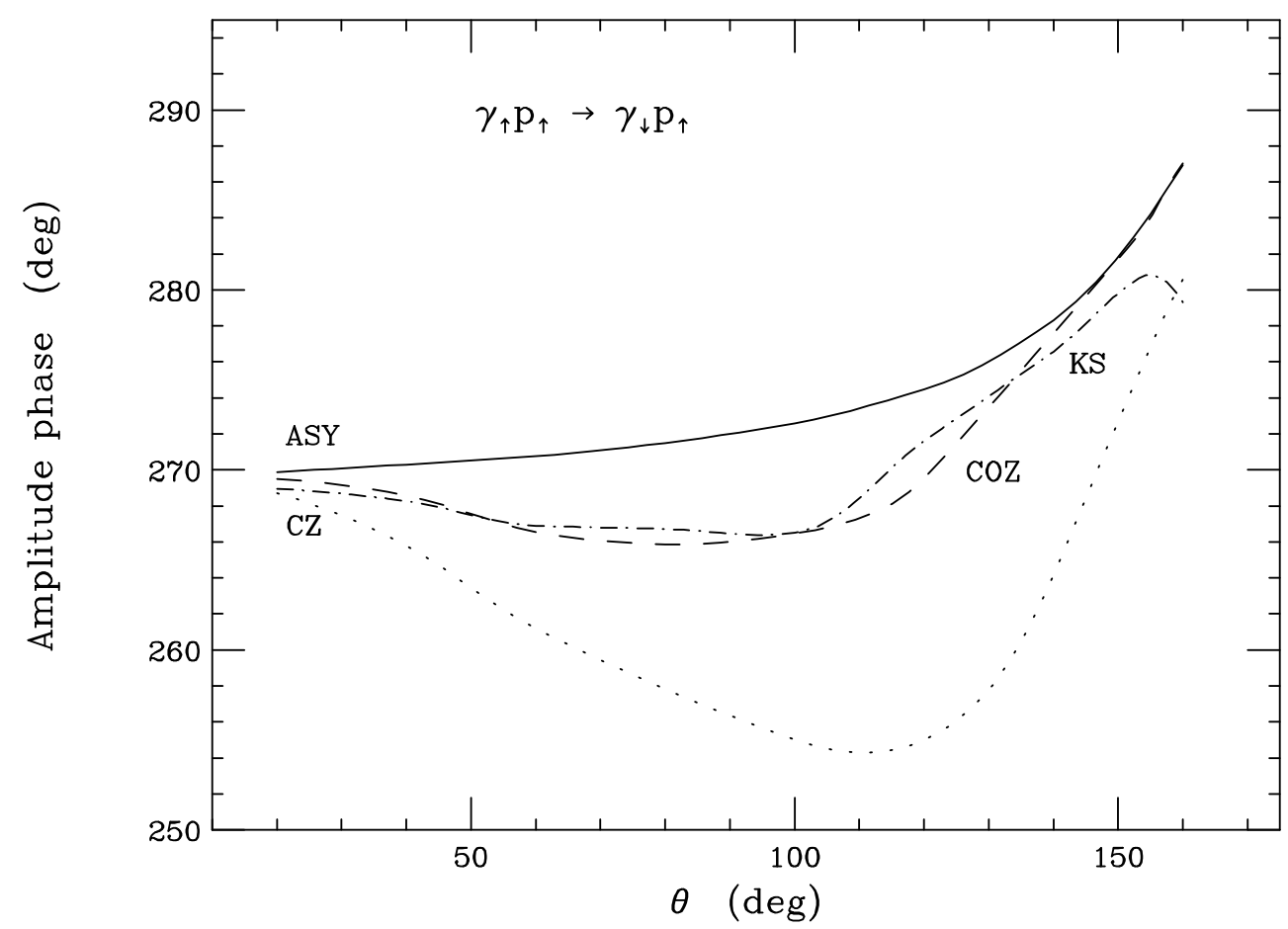

Figure 6: Phase of the helicity amplitude for $\gamma_{\uparrow} p_{\uparrow} \rightarrow \gamma_{\downarrow} p_{\uparrow}$.

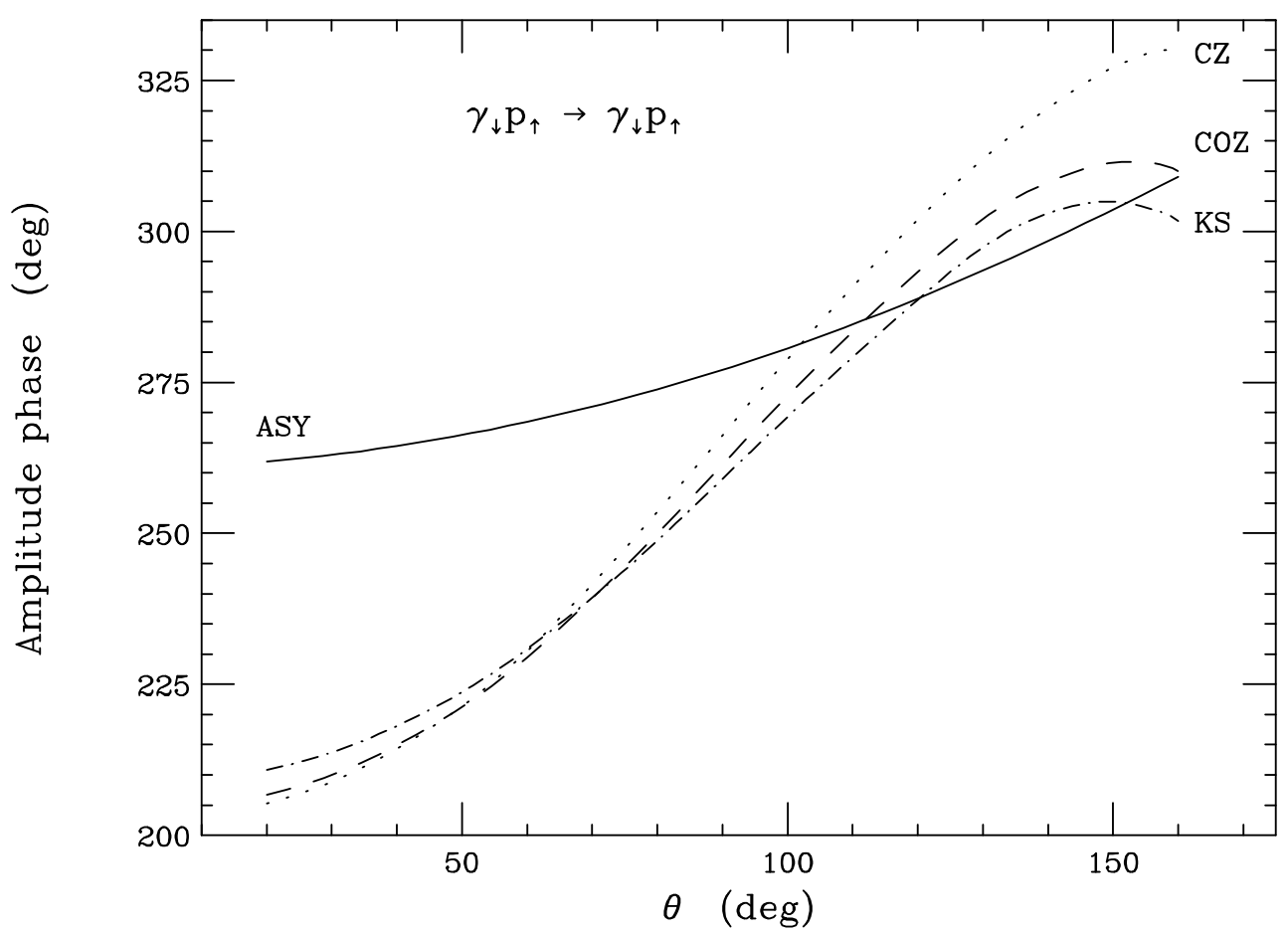

Figure 7: Phase of the helicity amplitude for $\gamma_{\downarrow} p_{\uparrow} \rightarrow \gamma_{\downarrow} p_{\uparrow}$. 


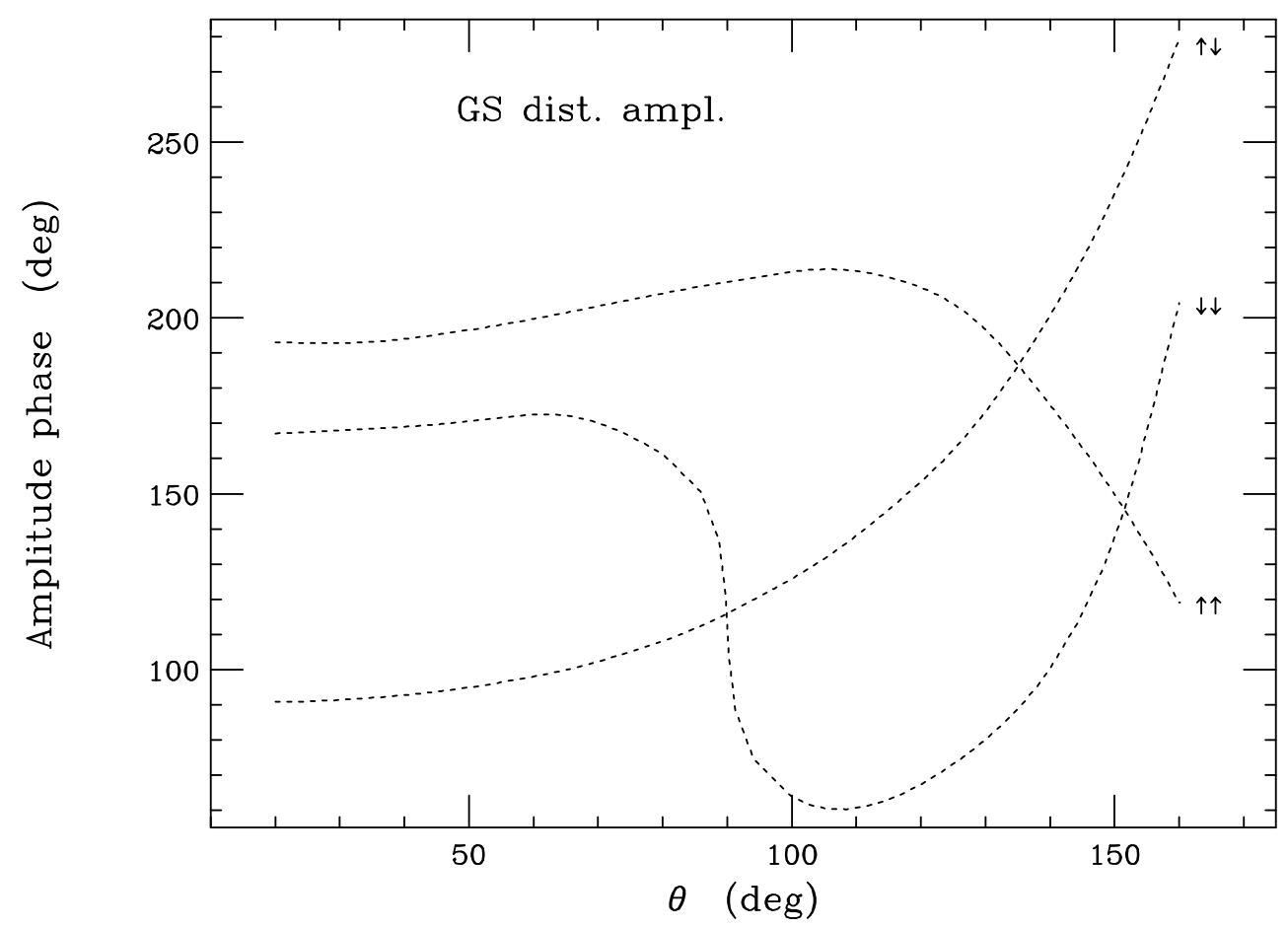

Figure 8: Phase of the three independent helicity amplitudes for the GS distribution amplitude. The arrows correspond to the photon helicities $\lambda, \lambda^{\prime}$ in the amplitudes $\mathcal{M}_{\uparrow \uparrow}^{\lambda \lambda^{\prime}}$. 


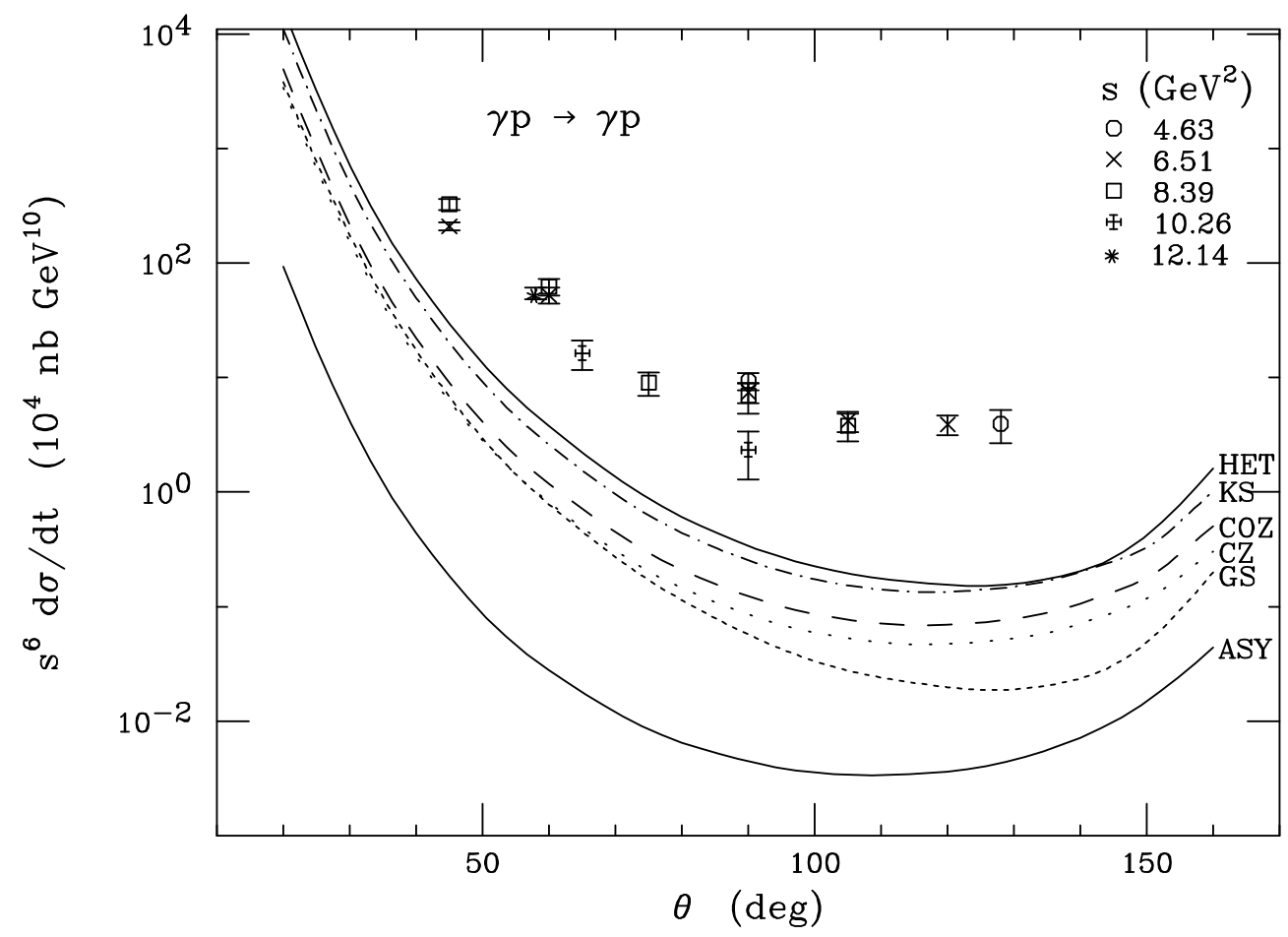

Figure 9: The unpolarized scaled cross section (12) for all six distribution amplitudes, for $\alpha_{s}=0.3$ and $f_{N}=5.2 \times 10^{-3} \mathrm{GeV}^{2}$, compared with experiment [20]. 


\begin{tabular}{|c|c|c|}
\hline$\theta(\mathrm{deg})$ & $10^{3} s^{2} \operatorname{Re}\left(\mathcal{M}_{\uparrow \uparrow}^{\uparrow \uparrow}\right)$ & $10^{3} s^{2} \operatorname{Im}\left(\mathcal{M}_{\uparrow \uparrow}^{\uparrow \uparrow}\right)$ \\
\hline 20 & $-74920 \pm 240$ & $29200 \pm 230$ \\
30 & $-15720 \pm 110$ & $5133 \pm 46$ \\
40 & $-5255 \pm 15$ & $1301 \pm 14$ \\
50 & $-2371.2 \pm 8.0$ & $348.6 \pm 5.8$ \\
60 & $-1273.6 \pm 4.3$ & $42.2 \pm 3.5$ \\
70 & $-768.8 \pm 2.3$ & $-72.1 \pm 2.3$ \\
80 & $-511.2 \pm 3.1$ & $-115.4 \pm 1.4$ \\
90 & $-369.8 \pm 1.2$ & $-139.0 \pm 1.0$ \\
100 & $-278.3 \pm 1.0$ & $-152.03 \pm 0.91$ \\
110 & $-222.4 \pm 1.2$ & $-165.53 \pm 0.90$ \\
120 & $-179.54 \pm 0.70$ & $-183.15 \pm 0.95$ \\
130 & $-144.2 \pm 1.0$ & $-211.6 \pm 1.0$ \\
140 & $-107.80 \pm 0.91$ & $-257.1 \pm 1.3$ \\
150 & $-52.9 \pm 2.7$ & $-324.9 \pm 2.6$ \\
160 & $75.9 \pm 3.1$ & $-415.5 \pm 5.0$ \\
\hline
\end{tabular}

Table 1: The real and imaginary parts of the helicity amplitude $\mathcal{M}_{\uparrow \uparrow}^{\uparrow \uparrow}$ for the COZ distribution amplitude (multiplied by $s^{2}$ in units of $\mathrm{GeV}^{4}$ ). The errors are from the VEGAS numerical integration. The values used for $f_{N}, \alpha_{\mathrm{em}}$, and $\alpha_{s}$ are the same as in the rest of the paper. The normalization is the same as in Table V of ref. [18] (which we found quite useful).

the PQCD cross section scales like $\alpha_{s}^{4}$, accommodating a factor of 10 by changing $\alpha_{s}$ would require $\alpha_{s} \approx 0.5$. While this is not out of the question, and while some variation in $f_{N}$ could be considered as well, this may be pushing the validity of perturbation theory. On the other hand, the shape of the curves (i.e., ignoring the overall normalization) matches the data quite well for the KS, COZ, CZ, and HET distribution amplitudes.

\subsection{Normalization by $F_{1}^{p}\left(Q^{2}\right)$}

As mentioned in the introduction, the $\alpha_{s}^{4}(\mu)$ scaling of the proton Compton cross section at Born level introduces a large normalization uncertainty into the PQCD prediction. Uncertainty in $f_{N}$ also contributes. Both of these uncertainties can be removed at Born level by considering the dimensionless ratio 29]

$$
\frac{s^{6} \frac{d \sigma_{\gamma p}}{d t}}{\left[Q^{4} F_{1}^{p}\left(Q^{2}\right)\right]^{2}},
$$

where $F_{1}^{p}\left(Q^{2}\right)$ is the elastic Dirac form factor for the proton at space-like momentum transfer $Q$. One might also imagine normalizing the Compton cross section by the time-like proton form factor. At leading order in $\alpha_{s}$, the PQCD predictions in the space-like and time-like regions are identical [1]; however, experimentally the time-like form factor is larger by a factor of about two [30, 31]. Higher order PQCD corrections can in principle account for 
this factor, as Sudakov effects are different in the two regions [32]. The Compton scattering kinematics are much closer to those of the space-like proton form factor than the time-like one, at least as far as the proton is concerned. Therefore Sudakov and related higher-order effects are best cancelled by normalizing with the space-like form factor.

At leading twist, $F_{1}^{p}\left(Q^{2}\right)$ is predicted to be the same as the magnetic form factor $G_{M}^{p}\left(Q^{2}\right)$. Experimentally, these are close but not identical [31]. To normalize the experimental Compton points, we use the experimental form factor values,

$$
Q^{4} F_{1}^{p}\left(Q^{2}\right) \approx Q^{4} G_{M}^{p}\left(Q^{2}\right) \approx 1.0 \mathrm{GeV}^{4}, \quad Q^{2} \approx 7-15 \mathrm{GeV}^{2}
$$

which are representative of the region where both scaled form factors flatten out, and are also similar to the highest experimental values of $s$ available in Compton scattering. f $^{\text {To }}$ normalize the theoretical Compton curves, we recalculated the proton form factor at leading order in PQCD, obtaining

$$
Q^{4} F_{1}^{p}\left(Q^{2}\right)=\frac{\left(4 \pi \alpha_{s} f_{N}\right)^{2}}{216} I_{F}
$$

where

$$
I_{F}= \begin{cases}2.500 \times 10^{5} & (\mathrm{CZ}) \\ 2.505 \times 10^{5} & (\mathrm{GS}) \\ 3.653 \times 10^{5} & (\mathrm{KS}) \\ 2.897 \times 10^{5} & (\mathrm{COZ}) \\ 3.303 \times 10^{5} & (\mathrm{HET}) \\ 0 & (\mathrm{ASY})\end{cases}
$$

These results, using the wave function (2) which is equivalent to that in ref. [10], are precisely a factor of two smaller than several previous calculations using the same wave functions [33]. We do not understand the origin of this discrepancy. We do agree with the normalization of the hard scattering amplitude and the form factor in ref. [34] (which uses, however, a different representation of the proton wave function than eq. (2)).

Figure 10 shows the Compton cross section, normalized according to eq. (13), for both PQCD and the experimental data. We omit the ASY distribution amplitude, since the leading order ASY form factor vanishes. Compared with the conventionally normalized curves in fig. 9, the spread between the predictions of the three qualitatively similar distribution amplitudes, KS, COZ and CZ, has become much smaller. The theoretical curves also lie a factor of 2 to 5 closer to the data. However, they still fall about an order of magnitude below the data at the widest scattering angles. (The HET distribution amplitude does slightly better than this.) Thus it seems unlikely that the elastic proton form factor and the Compton scattering amplitude are both described by PQCD at presently accessible energies, unless there are large higher-order and process-dependent corrections.

\footnotetext{
§If one equates the four-momentum transfer to the proton in the two processes $-Q^{2}$ in the form factor and $-t$ in Compton scattering - then the corresponding Compton $s=2 Q^{2} /(1-\cos \theta)$ should actually be considerably bigger than $Q^{2}$. At $90^{\circ}$, for example, $s=2 Q^{2}$. Unfortunately, there are no experimental Compton data with $s$ this large (all have $-t<5.3 \mathrm{GeV}^{2}$ ), so there is not a good overlap with the region (14) where the elastic form factor is beginning to scale properly.
} 


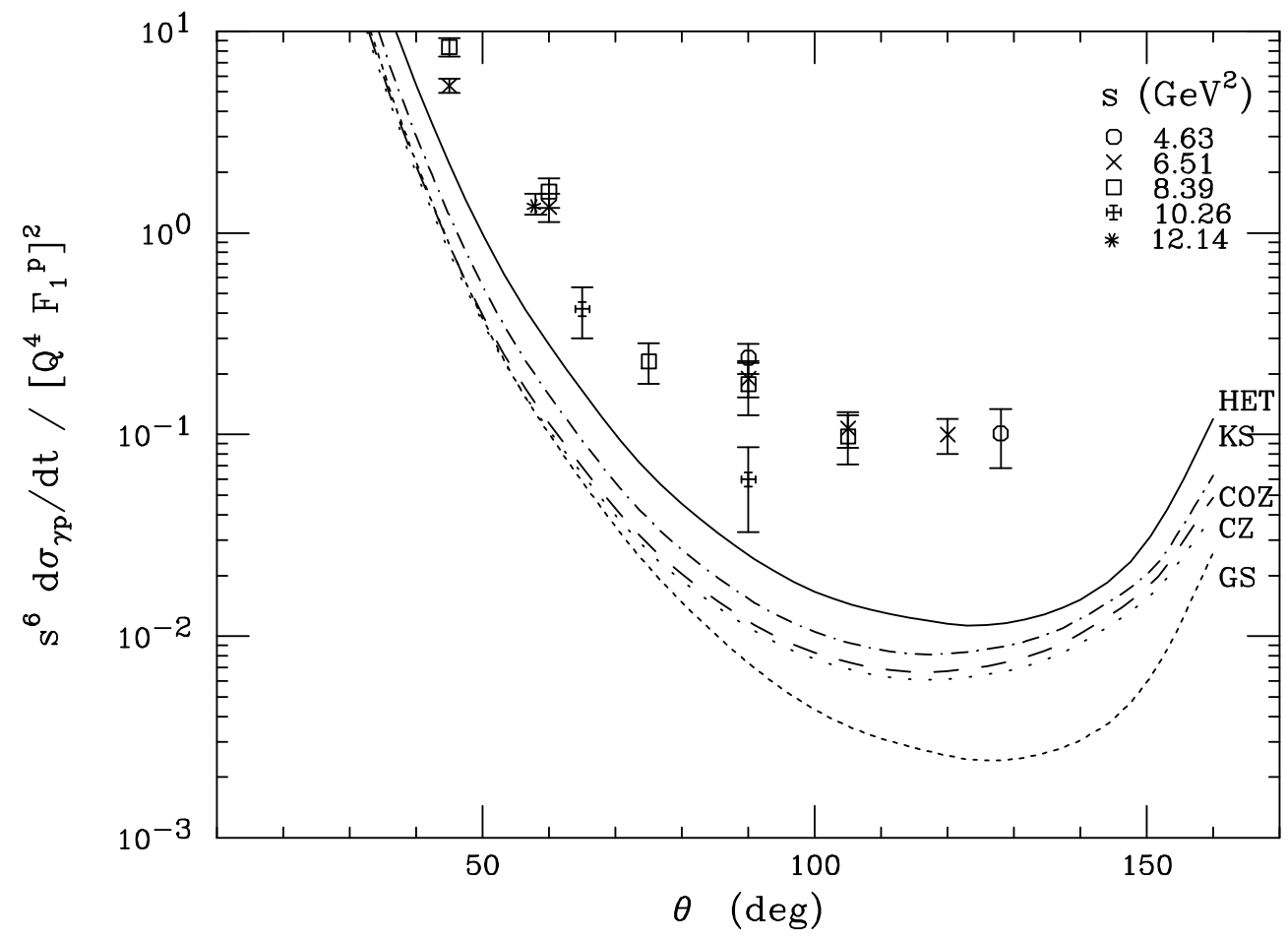

Figure 10: The scaled unpolarized Compton cross section, normalized by the scaled elastic proton form factor, as in eq. (13), for five distribution amplitudes, compared with the experimental data [20, 31. 


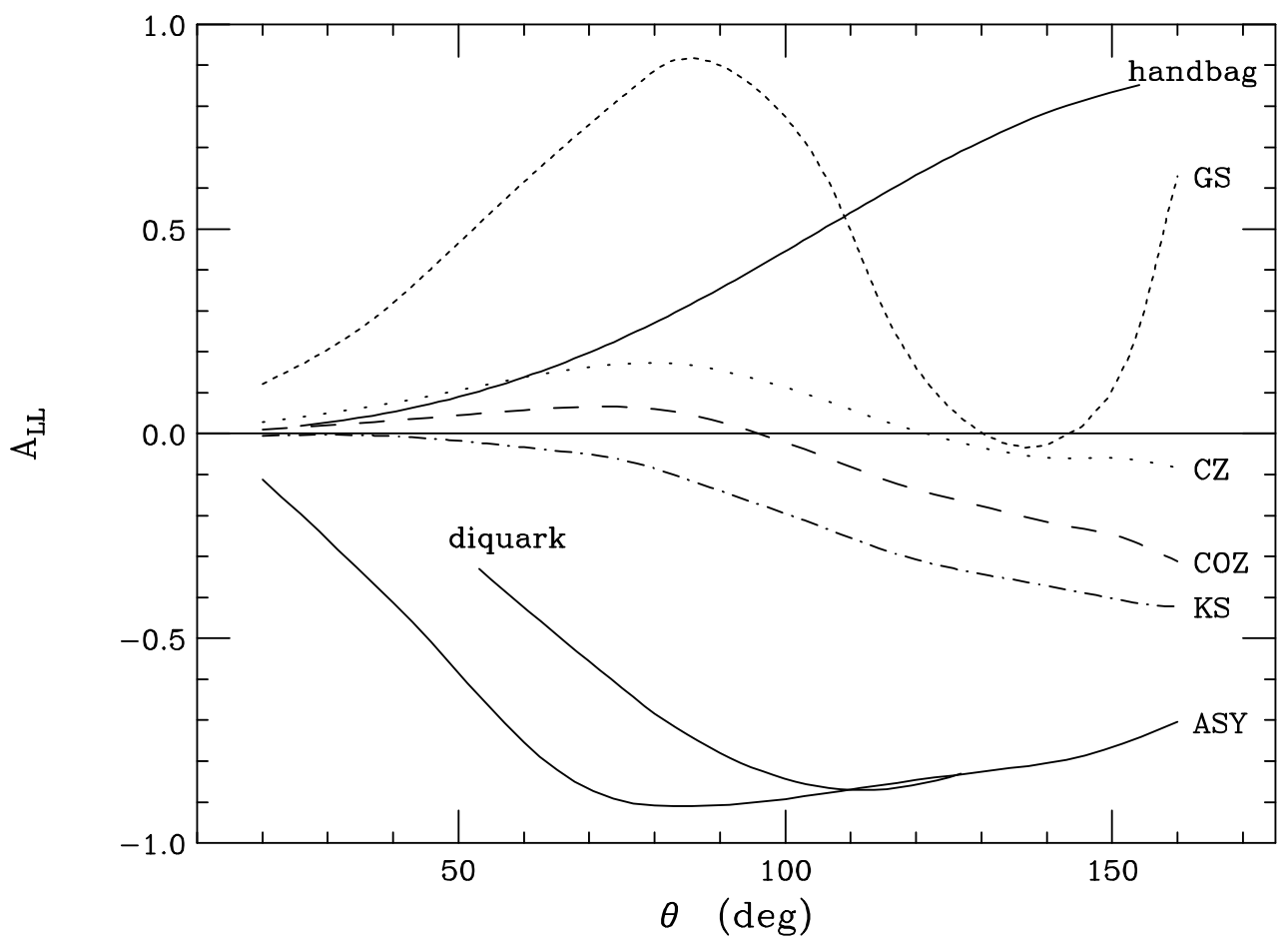

Figure 11: The initial state helicity correlation $A_{L L}$ in perturbative QCD for five distribution amplitudes. Also plotted is the handbag model prediction for $E_{\gamma}=4 \mathrm{GeV}$ (GRV) [任, and a diquark model prediction [5].

\subsection{Asymmetries}

Various polarization asymmetries can be constructed from the helicity amplitudes. These observables may provide additional diagnostic power for uncovering the Compton scattering mechanism, beyond what the unpolarized cross section provides.

Figure 11 presents the perturbative QCD results for the initial state helicity correlation $A_{L L}$ defined in eq. (10). Also shown is the handbag model prediction [4 for $E_{\gamma}=4 \mathrm{GeV}$, where the form factors $R_{V, A}$ were evaluated using the parton distribution functions of GRV [35]. In leading-twist PQCD, the proton helicity is conserved. The handbag model does not inherently require proton helicity conservation, but it has been assumed in ref. [4]. Thus the PQCD and handbag curves for $A_{L L}$ in fig. 11 can be equated to the longitudinal photon-to-proton polarization transfer asymmetry, which is slated to be measured for at least one scattering angle in an upcoming experiment [21]. The diquark model analyzed in ref. [5, 6] has nonvanishing proton helicity-flip amplitudes at finite $s$, making $A_{L L}$ and the polarization transfer into distinct asymmetries. We plot the diquark prediction for $A_{L L}$ from ref. [5]. Figure 11 shows that PQCD gives quite different qualitative behavior from both the handbag and diquark models for $A_{L L}$, and they should be distinguishable with the help of experimental data at just a couple of backward scattering angles. A caveat is that the GS curve is somewhat oscillatory, so one might wonder whether a distribution amplitude 


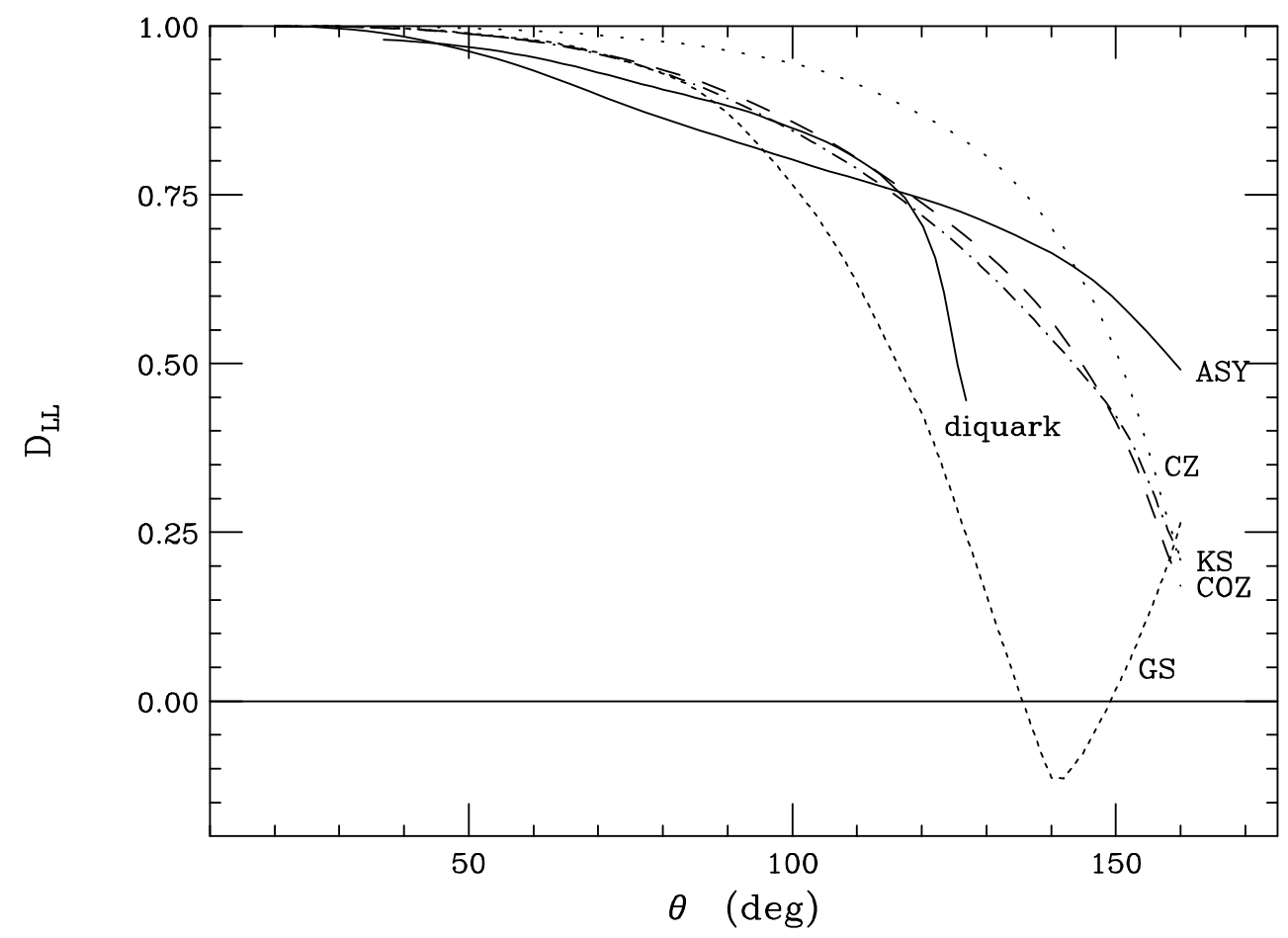

Figure 12: The photon spin transfer coefficient $D_{L L}$ in perturbative QCD for five distribution amplitudes. Also plotted is the diquark model prediction for $E_{\gamma}=4 \mathrm{GeV}$ (standard DA) [6]. The handbag model predicts $D_{L L}=1$.

'between' GS and the $\{\mathrm{CZ}, \mathrm{COZ}, \mathrm{KS}\}$ class of amplitudes could produce behavior similar to the handbag model.

One can also define [6] a photon spin transfer coefficient

$$
D_{L L} \equiv \frac{\frac{d \sigma^{++}}{d t}-\frac{d \sigma^{+-}}{d t}}{\frac{d \sigma^{++}}{d t}+\frac{d \sigma^{+-}}{d t}},
$$

where now $d \sigma^{\lambda \lambda^{\prime}} / d t$ is the differential cross section for initial and final state photon helicities $\lambda$ and $\lambda^{\prime}$, and unpolarized incoming and outgoing protons. Figure 12 gives the PQCD predictions for this asymmetry, as well as that of the diquark model for $E_{\gamma}=4 \mathrm{GeV}$ and a 'standard' distribution amplitude [6]. The handbag model predicts $D_{L L}=1$, basically because the helicity-flip quark Compton amplitude $\gamma_{\uparrow} q \rightarrow \gamma_{\downarrow} q$ vanishes at Born level for massless quarks.

The final asymmetry we plot is the photon asymmetry [6]

$$
\Sigma \equiv \frac{\frac{d \sigma_{\perp}}{d t}-\frac{d \sigma_{\|}}{d t}}{\frac{d \sigma_{\perp}}{d t}+\frac{d \sigma_{\|}}{d t}}
$$




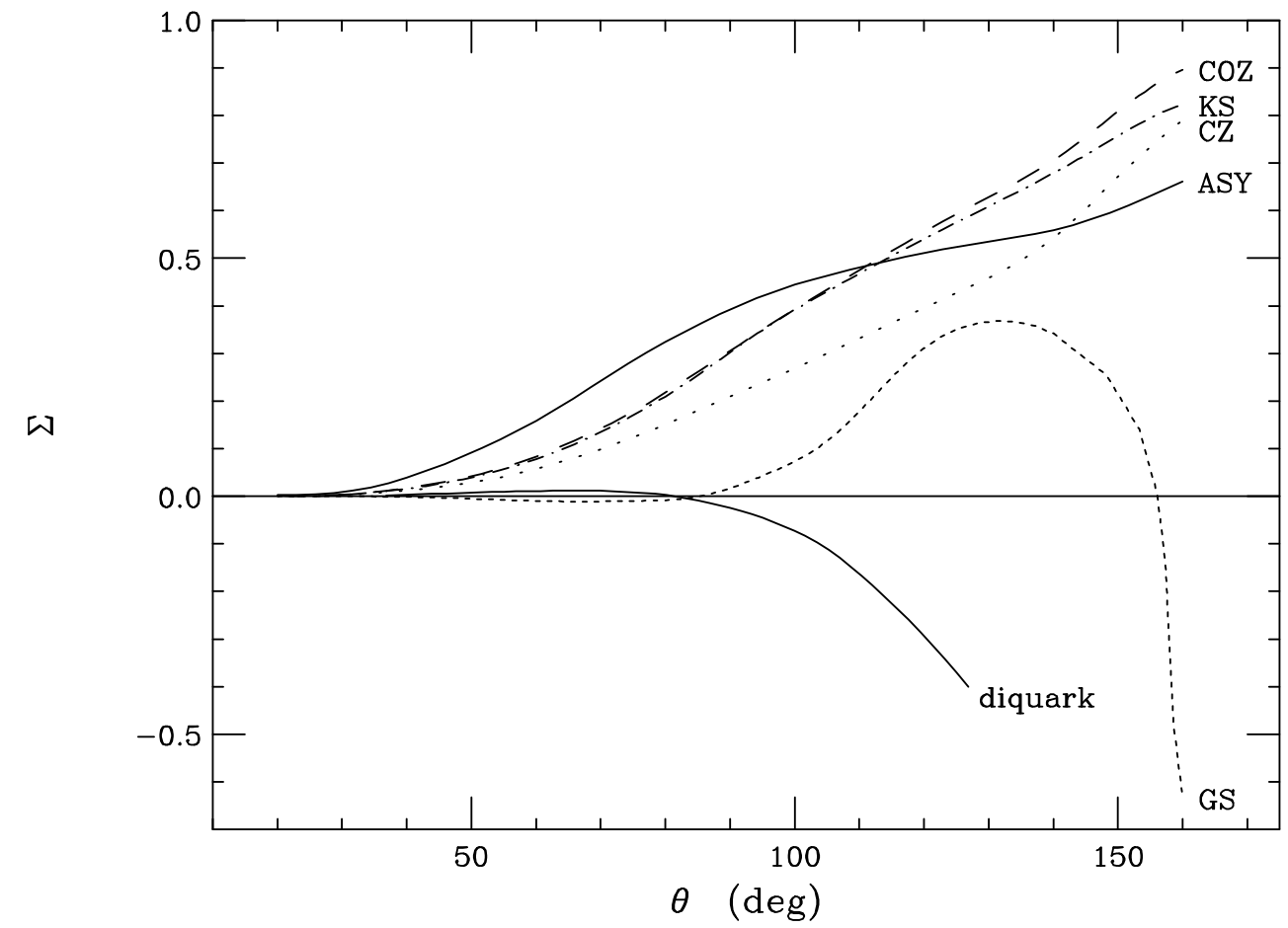

Figure 13: The photon asymmetry $\Sigma$ in perturbative QCD for five distribution amplitudes. Also plotted is the diquark model prediction for $E_{\gamma}=4 \mathrm{GeV}$ (standard DA) [6]. The handbag model predicts $\Sigma=0$. 
where $d \sigma_{\perp} / d t$ and $d \sigma_{\|} / d t$ are the differential cross sections for linearly polarized photons, with the polarization plane perpendicular or parallel (respectively) to the scattering plane. Generation of this asymmetry requires a nonzero photon helicity-flip amplitude; hence the asymmetry vanishes in the handbag model. Figure 13 plots the PQCD and diquark predictions. The diquark prediction is shown for $E_{\gamma}=4 \mathrm{GeV}$ and a 'standard' distribution amplitude; for another distribution amplitude $\Sigma$ can become positive in the backward region instead of negative [6]. This asymmetry has actually been measured [36], however only for $E_{\gamma}=3.45 \mathrm{GeV}$ and $\cos \theta>0.8$. A high-energy wide-angle measurement would be very useful for distinguishing between handbag and PQCD mechanisms.

\section{Conclusions}

Motivated by conflicting results in the literature, we have recalculated the fixed-order, Born level predictions of perturbative QCD for proton Compton scattering, for five different distribution amplitudes. While our results do not agree with those of any previous group, they do agree very well with those of ref. [19] for $\theta<110^{\circ}$, and the differences for $\theta>110^{\circ}$ seem to be dominated by a single helicity amplitude, $\mathcal{M}_{\uparrow \uparrow}^{\uparrow}$.

From the helicity amplitudes we computed three separate polarization asymmetries. Experimental measurements of these asymmetries could be used in conjunction with the unpolarized differential cross section in order to help shed light on the mechanism involved in the Compton scattering process.

We also have attempted to reduce the uncertainty in the overall normalization of the Compton cross section by normalizing it by the square of the elastic proton form factor. This exercise reduces the spread in the theoretical predictions, but it leaves them an order of magnitude below the data. Unfortunately, this result makes it difficult to simultaneously explain the current data on the elastic proton form factor and on Compton scattering in terms of perturbative QCD, without appealing to large uncalculated higher-order and processdependent corrections.

\section{Acknowledgments}

We thank Stan Brodsky for suggesting this work, and for useful discussions and comments on the manuscript. We are grateful to Marc Vanderhaeghen and particularly Andreas Kronfeld for detailed discussions of their work and for providing us with unpublished data files. We thank Markus Diehl for several helpful remarks and suggestions.

\section{References}

[1] G.P. Lepage and S.J. Brodsky, Phys. Rev. D22, 2157 (1980).

[2] A.V. Radyushkin, Phys. Rev. D58, 114008 (1998) [hep-ph/9803316].

[3] M. Diehl, T. Feldmann, R. Jakob and P. Kroll, Eur. Phys. J. C8, 409 (1999) hep$\mathrm{ph} / 9811253$. 
[4] M. Diehl, T. Feldmann, R. Jakob and P. Kroll, Phys. Lett. B460, 204 (1999) hepph/9903268.

[5] P. Kroll, M. Schurmann and W. Schweiger, Int. J. Mod. Phys. A6, 4107 (1991).

[6] C.F. Berger, Diplomarbeit, Technical University of Graz (November 1997).

[7] V.L. Chernyak and I.R. Zhitnitsky, Nucl. Phys. B246, 52 (1984).

[8] M. Gari and N.G. Stefanis, Phys. Lett. B175, 462 (1986);

Phys. Rev. D35, 1074 (1987).

[9] I.D. King and C.T. Sachrajda, Nucl. Phys. B279, 785 (1987).

[10] V.L. Chernyak, A.A. Oglobin and I.R. Zhitnitsky, Yad. Fiz. 48, 841 (1988) [Sov. J. Nucl. Phys. 48, 536 (1988)]; Z. Phys. C42, 569 (1989).

[11] N.G. Stefanis and M. Bergmann, Phys. Rev. D47, R3685 (1993) hep-ph/9211250.

[12] M. Bergmann and N.G. Stefanis, Phys. Lett. B325, 183 (1994) hep-ph/9403209.

[13] R. Eckardt, J. Hansper and M.F. Gari, Phys. Rev. D50, 26 (1994); hep-ph/9607380.

[14] N. Isgur and C.H. Llewellyn Smith, Phys. Lett. B217, 535 (1989).

[15] J. Bolz and P. Kroll, Z. Phys. A356, 327 (1996) hep-ph/9603289.

[16] E. Maina and G.R. Farrar, Phys. Lett. B206, 120 (1988).

[17] G.R. Farrar and H. Zhang, Phys. Rev. D41, 3348 (1990), D42, 2413(E) (1990); Phys. Rev. Lett. 65, 1721 (1990).

[18] A.S. Kronfeld and B. Nizic, Phys. Rev. D44, 3445 (1991), D46, 2272(E) (1992).

[19] M. Vanderhaeghen, P.A. Guichon and J. Van de Wiele, DAPNIA-SPHN-96-36, Presented at Workshop on Virtual Compton Scattering, Clermont-Ferrand, France, 26-29 Jun 1996; Nucl. Phys. A622, 144c (1997);

P.A. Guichon and M. Vanderhaeghen, Prog. Part. Nucl. Phys. 41, 125 (1998) hepph/9806305.

[20] M.A. Shupe et al., Phys. Rev. D19, 1921 (1979).

[21] A.M. Nathan, hep-ph/9908522;

J.P. Chen et al. [Hall A Collaboration], PCCF-RI-99-17.

[22] See e.g. http://www.cebaf.gov/div_dept/physics_division/GeV.html. 
[23] ELFE "blue book", J.-M. DeConto, ed., Institute des Sciences Nucléaires de Grenoble, RME02 (October 1993);

R. Brinkmann et al., Nucl. Phys. A622, 187c (1997);

P. Hoyer, Nucl. Phys. A622, 284C (1997) hep-ph/9703462; hep-ph/9911486.

[24] G.R. Farrar and F. Neri, Phys. Lett. 130B, 109 (1983), 152B, 443(E) (1985).

[25] M. Vanderhaeghen, private communication.

[26] A.S. Kronfeld, private communication.

[27] G.P. Lepage, J. Comput. Phys. 27, 192 (1978).

[28] W.H. Press, S.A. Teukolsky, W.T. Vetterling and B.P. Flannery, Numerical Recipes in C: The art of scientific computing (Cambridge Univ. Press, 1996).

[29] S.J. Brodsky, private communication.

[30] M. Ambrogiani et al. [E835 Collaboration], Phys. Rev. D60, 032002 (1999).

[31] A. F. Sill et al., Phys. Rev. D48, 29 (1993).

[32] T. Gousset and B. Pire, Phys. Rev. D51, 15 (1995) hep-ph/9403293.

[33] V. L. Chernyak, A. A. Ogloblin and I. R. Zhitnitsky, Z. Phys. C42, 583 (1989);

C. Ji, A. F. Sill and R. M. Lombard, Phys. Rev. D36, 165 (1987);

N. G. Stefanis, Phys. Rev. D40, 2305 (1989).

[34] C. E. Carlson and F. Gross, Phys. Rev. D36, 2060 (1987).

[35] M. Gluck, E. Reya and A. Vogt, Z. Phys. C67, 433 (1995);

M. Gluck, E. Reya, M. Stratmann and W. Vogelsang, Phys. Rev. D53, 4775 (1996) hep-ph/9508347.

[36] G. Buschhorn et al., Phys. Lett. 37B, 211 (1971). 